\title{
High food insecurity in Latinx families and associated COVID-19 infection in the Greater Bay Area, California
}

Milagro Escobar, Andrea DeCastro Mendez, Maria Romero Encinas, Sofia Villagomez and Janet M. Wojcicki

\begin{abstract}
Background: Food insecurity impacts nearly one-in-four Latinx households in the United States and has been exacerbated by the novel coronavirus or COVID-19 pandemic.

Methods: We examined the impact of COVID-19 on household and child food security in three preexisting, longitudinal, Latinx urban cohorts in the San Francisco Bay Area ( $N=375$ households, 1875 individuals). Households were initially recruited during pregnancy and postpartum at Zuckerberg San Francisco General Hospital (ZSFG) and UCSF Benioff prior to the COVID-19 pandemic. For this COVID-19 sub-study, participants responded to a 15-min telephonic interview. Participants answered 18 questions from the US Food Security Food Module (US HFSSM) and questions on types of food consumption, housing and employment status, and history of COVID-19 infection as per community or hospital-based testing. Food security and insecurity levels were compared with prior year metrics.

Results: We found low levels of household food security in Latinx families (by cohort: 29.2\%; 34.2\%; 60.0\%) and child food security $(56.9 \%, 54.1 \%, 78.0 \%)$ with differences between cohorts explained by self-reported levels of education and employment status. Food security levels were much lower than those reported previously in two cohorts where data had been recorded from prior years. Reported history of COVID-19 infection in households was $4.8 \%$ (95\% Confidence Interval (Cl); $1.5-14.3 \%) ; 7.2 \%(95 \% \mathrm{Cl}, 3.6-13.9 \%)$ and 3.5\% (95\%Cl, 1.7-7.2\%) by cohort and was associated with food insecurity in the two larger cohorts $(p=0.03 ; p=0.01$ respectively).

Conclusions: Latinx families in the Bay Area with children are experiencing a sharp rise in food insecurity levels during the COVID-19 epidemic. Food insecurity, similar to other indices of poverty, is associated with increased risk for COVID-19 infection. Comprehensive interventions are needed to address food insecurity in Latinx populations and further studies are needed to better assess independent associations between household food insecurity, poor nutritional health and risk of COVID-19 infection.
\end{abstract}

Keywords: COVID-19, Latinx, Food insecurity, Children

\footnotetext{
* Correspondence: Janet.wojcicki@ucsf.edu

Division of Pediatric Gastroenterology, Hepatology and Nutrition, University

of California, 550 16th Street, 5th Floor, San Francisco, CA 94158-0136, USA
} 


\section{Background}

Food insecurity is common among Latinx families, the largest ethnic minority group in the United States, and has been further exacerbated by the novel coronavirus pandemic (COVID-19) [1]. Prior to COVID-19, 1 in 9 households faced food insecurity with a higher prevalence in Latinx families $[2,3]$. Latinx individuals comprise over 60 million individuals in the United States [4] and are twice as likely as White Americans to face poverty including aspects of food insecurity [5]. Preliminary reports since the beginning of the COVID-19 shelter-in-place lockdowns in the United States (approximately March 2020) suggest that Latinx families have had higher increases in food insecurity compared to Whites (32\% versus $18 \%)$ with even higher rates in families with children [6].

Higher rates of food insecurity in Latinx communities can be explained by sociodemographic and economic factors specific to these communities. Latinx individuals are more likely to be low-income, recent immigrants, have young children, live in larger households, and reside in urban areas that have been historically prone to food insecurity [7-10]. Furthermore, Latinx adults may have been most impacted by loss of employment due to sectors that were particularly impacted by COVID-19 shutdowns including the hotel industry, food services, healthcare, and manufacturing [11]. Similar to other high-risk groups, Latinx families have relied on government food services since the start of the pandemic [12]. However, the number of Latinx families served is likely underestimated as many Latinx immigrants who contribute to the US economy are not able or resistant to receiving economic relief in context of the COVID-19 pandemic due to lack of legal status. Additional disparities in Latinx communities include crowded living conditions, housing insecurity, education gaps, few family resources, all which create higher risk for COVID-19 infection [13].

We have previously assessed the prevalence of food insecurity in Latinx low-income families residing in six counties of the San Francisco Bay Area finding high rates prior to COVID-19 [14]. Slightly more than half of the households previously surveyed had high food security (58.3\%) whereas the remainder experienced marginal food security (8.6\%), low food security (28.2\%), and very low food security (4.9\%). Food insecurity in children has been linked to lower academic performance, anxiety, obesity and diabetes. Adults with food insecurity can have lower scores on mental and physical health assessments higher risk of depression and anxiety, chronic disease, and poorer overall health status. Previous studies have also suggested that food insecurity may increase risk for infectious disease such as HIV in the context of exposure due to poor immunological health [15].
To our knowledge, no study has investigated the impact of COVID-19 on food security in a longitudinal, Latinx sample of participants residing in an urban U.S. context. We examined food security during COVID-19 using three prospective longitudinal studies of Latinx households based in the 6 counties of the San Francisco Bay Area. We evaluated levels of food security and insecurity during COVID-19 compared with previous levels (prior to COVID-19) in these populations as well as the association between COVID-19 infection and food insecurity.

\section{Methods}

\section{Recruitment and consent}

Mothers were recruited from three previous longitudinal birth cohort studies: the Hispanic Eating and Nutrition Study (HEN) $(n=201)[16,17]$, Latinx, Eating, and Diabetes Study (LEAD) $(n=97)[18,19]$ and the Telomeres At Birth Study (TAB) $(n=424)$. Participants for both HEN and LEAD were recruited during pregnancy as previously described and $\mathrm{TAB}$ participants were recruited post-partum before being discharged from the hospital. All participants from HEN, LEAD and TAB have been followed up over the course of approximately 13,8 , and 1-2 years respectively. These cohorts were initially recruited to evaluate the relationship between prenatal and early postnatal exposures and development of childhood obesity. Inclusion criteria for HEN and LEAD included self-identifying as Latinx and expecting a healthy newborn infant as previously described. Participants for HEN and LEAD were primarily recruited from Zuckerberg San Francisco General Hospital (ZSFG) with a smaller percentage from UCSF Benioff for $\operatorname{HEN~}[17,19]$. These two cohorts consist of families of primarily Spanish-speaking foreign-born adults with less than a high school education and originating primarily from the countries of Central America and Mexico [17, 19]. We initially recruited all race/ethnic backgrounds for $\mathrm{TAB}$ but restricted entry criteria to Latinx for the second phase of our study. TAB participants were recruited from UCSF Benioff (70.2\%) and ZSFG (29.8\%) and represent a broader background of education levels, countries of ancestry and place of birth.

To assess participation in the COVID-19 food insecurity sub-study, participants were contacted by telephone during the months of May to September 2020 using previous contact information. Less than $10 \%$ of those that were successfully contacted declined participation, although we were not able to contact a significant percentage of our cohorts due to a limited time period of recruitment. Participants were asked for their language preference (English or Spanish) and were provided information about the COVID-19 sub-study by bilingual research coordinators and the study principal investigator. 
We obtained verbal consent from those participants who were interested, and participants took part in a 15minute interview by telephone. Food insecurity was assessed using the US Household Food Security Scale Module (US HFSSM) that we have used annually with HEN and LEAD studies to assess food insecurity [20]. The US HFSSM has been validated for use in Spanish with low -income Spanish speaking adults from Mexico, Central America, Cuba and Puerto Rico [21] and has also been confirmed for face validity through qualitative focus groups with low income pregnant and postpartum Latinx women in the United States [22].

We additionally asked questions about COVID-19 symptoms and testing, housing and employment status and food consumption. Participants were compensated for their time for participation with a \$15 gift card to Target or Amazon based on participant preference. The principal investigator of the study (JW) and four research coordinators including (ME, ADC and MR) conducted all interviews. The Committee on Human Research (CHR), the UCSF Institutional Review Board approved all aspects of the study (IRB numbers 1106334; 16-06163; 16-18535).

\section{Procedures}

Food insecurity was measured using the 18-item US Household Food Security Scale Module. The timeframe of the questions was modified to collect data on food access from when COVID-19 lockdowns first began (March 2020) to the time of interview. The Food Insecurity Module included questions regarding adult and children's eating habits [20]. Scoring was done automatically by REDCap, with higher scores indicating a higher level of food insecurity. We followed previously established food security categories: high food security or food secure (0 affirmative responses), marginal food security (1-2 affirmative responses), low food security (37 affirmative responses), and very low food security (818 affirmative responses). Child food security levels were categorized as high food security or food secure (0 affirmative responses), marginal food security (1 affirmative response), low food security (2-4 affirmative response) and very low food security (5-8 affirmative responses). We compared mean \pm SD food security scores and categorical markers of food security with prior year assessments of food security in two out of three cohorts (HEN and LEAD). We had no prior assessment of food security in TAB participants. Previous levels of household and child food security collected in the years before the COVID-19 pandemic were compaed with food security levels during the pandemic.

Food assistance information was collected prior to the COVID-19 pandemic as part of our previous longitudinal data collection (self-reported participation in the
Special Supplemental Program for Women, Infants and Children's program (WIC) or participation in the Supplemental Nutrition Assistance Program (SNAP; previously known as food stamps)). Similarly, maternal education level (primary or less than a high school diploma, high school diploma, some college or more) and primary language use (Spanish, English or bilingual) was also collected at study entry for all participants. As part of the COVID-19 sub-study we asked about adult household employment status, number of total household members as well as crowding indices including size of house (number of bathrooms and bedrooms). As the COVID-19 sub-study was a new part of our overall study, we pilot tested the face validity of the questions with the first 10 participants, making subtle initial changes to the questionnaire asking participants to repeat or rephrase their understanding of what the question was asking.

Participants were also asked about COVID-19 symptoms, laboratory-based testing as well as duration of symptoms for all household members since middle of March 2020 until time of interview. Household units were the level of the analysis.

\section{Statistical analysis}

Household and child food security levels and corresponding insecurity were calculated including mean score \pm standard deviation (SD) as well as percentages by category. Data was stratified by cohort using Stata 15.0. Levels of household and child food security were assessed for normality using graphical assessments as well as statistical tests to assess normality including Shapiro-Wilk. As there were departures from normality, non-parametric statistical tests were used to assess associations including Wilcoxon rank sum, Kruskal-Wallis and Spearman rank order correlation coefficient for continuous predictors and chi-squared tests for categorical ones. Associations between household and child food insecurity levels (continuous measures and categorical variables) and education level (high school or lower versus more than high school education), language use (Spanish, English or bilingual), COVID-19 testing positivity, and employment status (unemployed or adult household employment) were assessed using non-parametric methods. Multivariable logistic models were used to evaluate independent predictors of household COVID infection in relation to food insecurity in TAB and HEN cohorts adjusting for potential confounders such as unemployment and crowding that were significant at $p$ value $<0.05$ in bivariate analysis. We did not assess independent predictors of household COVID infection in LEAD using modeling due to the small sample size of that cohort. 
Table 1 Demographics and Household Specifics by Bay Area Cohort

\begin{tabular}{|c|c|c|c|}
\hline \multirow[t]{4}{*}{ Cohort Name } & Telomeres at Birth (TAB) & Latinx, Eating and Diabetes (LEAD) & Hispanic, Eating and Nutrition (HEN) \\
\hline & $\%(N)$ & $\%(\mathrm{~N})$ & $\%(\mathrm{~N})$ \\
\hline & Mean \pm SD & Mean \pm SD & Mean \pm SD \\
\hline & $N=200$ families & $N=65$ families & $N=111$ families \\
\hline \multicolumn{4}{|l|}{ Household size } \\
\hline No. of people & $4.8 \pm 1.7$ & $5.3 \pm 2.0$ & $4.9 \pm 1.6$ \\
\hline No. of children & $2.4 \pm 1.1$ & $2.7 \pm 1.1$ & $2.9 \pm 1.2$ \\
\hline Number share bedroom & $2.5 \pm 1.0$ & $2.8 \pm 1.3$ & $2.3 \pm 1.1$ \\
\hline Number share bathroom & $3.3 \pm 1.6$ & $4.5 \pm 1.6$ & $3.8 \pm 1.6$ \\
\hline \multicolumn{4}{|l|}{ Food Assistance } \\
\hline SNAP/Food Stamps & Not Collected & $47.1 \%$ & $43.4 \%$ \\
\hline WIC participation & $41.4 \%$ & $15.7 \%$ & $72.9 \%$ \\
\hline \multicolumn{4}{|l|}{ Language Use } \\
\hline Spanish & $38.7 \%$ & $90.6 \%$ & $91.1 \%$ \\
\hline English & $56.8 \%$ & $4.7 \%$ & $8.9 \%$ \\
\hline Bilingual & $4.5 \%$ & $4.7 \%$ & - \\
\hline \multicolumn{4}{|l|}{ (English/Spanish) } \\
\hline \multicolumn{4}{|l|}{ Maternal Education } \\
\hline \multicolumn{4}{|l|}{ Highest Education Level } \\
\hline High School Or less & $34.3 \%$ & $79.4 \%$ & $75.2 \%$ \\
\hline \multicolumn{4}{|l|}{ Employment } \\
\hline All household adults unemployed & $24.1 \%$ & $35.2 \%$ & $38.8 \%$ \\
\hline
\end{tabular}

\section{Results}

We recruited 200 Latinx families from TAB (250 total recruited including 50 non-Latinx families), 111 from HEN and 64 from LEAD for a total participation of 375 Latinx families. Mean household size was $4.8 \pm 1.7$ for $\mathrm{TAB}, \quad 5.3 \pm 2.0$ for LEAD, and $4.9 \pm 1.6$ for HEN (Table 1). Total sample size of all household members for all three longitudinal studies combined was 1875. All studies reported a comparable number of children in households with a mean of $2.4 \pm 1.1$ for TAB, $2.7 \pm 1.1$ for LEAD and $2.9 \pm 1.2$ for HEN (Table 1).

Close to half of HEN and LEAD participants reported receiving SNAP benefits (food stamps) prior to the onset of the COVID-19 pandemic (43.4 and $47.1 \%$ respectively). We did not have this information on TAB participants. All of the HEN and LEAD participants selfidentified as Latinx with $80.6 \%$ of the TAB participants identifying as Latinx (13.8\% non-Hispanic Caucasian and $5.3 \%$ as Asian including Pacific Islander). We only included those TAB participants in this COVID-19 substudy who identified as Latinx $(n=200)$.

A high percentage of HEN and LEAD participants reported using Spanish as a primary language (91.1 and 90.5\%) with a much lower percentage reporting Spanish as the primarily language (38.7\%) for TAB participants.
Other sociodemographic differences between HEN, LEAD and $T A B$ included higher maternal education levels for TAB. Among TAB participants, $34.3 \%$ had a high school education or less $(65.7 \%$ with university or postgraduate qualifications) compared with $79.4 \%$ of LEAD reporting high school as the highest qualification $(20.6 \%$ with university/postgraduate) and $75.2 \%$ for HEN (24.8\% with university/postgraduate). During the COVID-19 pandemic time frame studied, $24.1 \%$ of TAB participants, $35.2 \%$ of LEAD and $38.8 \%$ of HEN households had no adults who were employed.

\section{Food security}

Two out of three cohorts report low rates of food security including LEAD (29.2\%) and HEN (34.2\%) compared to higher reports of household food security for $\mathrm{TAB}$ participants $(60 \%$, Table 2$)$. Similarly, a high percentage of participants in HEN and LEAD report low and very low food security (46.2\% for LEAD and $49.5 \%$ for HEN) with a much lower $26.5 \%$ for TAB. Slightly more than half of participants from HEN and LEAD report child food security. LEAD and HEN reported low levels of child food security (56.9 and $54.1 \%$ respectively) which was much higher for TAB (78\%) (Table 2). 
Table 2 Levels of Household, Child Food Security and COVD-19 Infection by Bay Area Cohort

\begin{tabular}{|c|c|c|c|}
\hline & Telomeres at Birth (TAB) & Latinx, Eating and Diabetes (LEAD) & Hispanic, Eating and Nutrition (HEN) \\
\hline & $\%(N)$ & $\%(N)$ & $\%(N)$ \\
\hline & Mean \pm SD & Mean \pm SD & Mean \pm SD \\
\hline & $N=200$ & $N=65$ & $N=111$ \\
\hline \multicolumn{4}{|l|}{ Levels of Household Food Security } \\
\hline Food Security Score, Mean \pm SD & $1.6 \pm 2.7$ & $3.3 \pm-3.7$ & $3.5 \pm 3.6$ \\
\hline High Food Security (Food Secure) & $60.0(120)$ & $29.2(19)$ & $34.2(38)$ \\
\hline Marginal Food Security & $13.5(27)$ & $24.6(16)$ & $16.2(18)$ \\
\hline Low Food Security & $20.5(41)$ & $33.9(22)$ & $31.5(35)$ \\
\hline Very Low Food Security & $6.0(12)$ & $12.3(8)$ & $18.0(20)$ \\
\hline \multicolumn{4}{|l|}{ Levels of Child Food Security } \\
\hline High Food Security (Food Secure) & $78.0(156)$ & $56.9(37)$ & $54.1(60)$ \\
\hline Marginal Food Security & $8.0(16)$ & $10.8(7)$ & $10.8(12)$ \\
\hline Low Food Security & $12.0(24)$ & $26.15(17)$ & $32.4(36)$ \\
\hline Very Low Food Security & $4(2.0)$ & $6.15(4)$ & $2.7(3)$ \\
\hline \multicolumn{4}{|l|}{ COVID Infection History } \\
\hline \multirow[t]{2}{*}{ Positive test reported March-Sept 2020} & $7 / 200(3.5 \%)$ & $3 / 62(4.8 \%)$ & 8/111 (7.2\%) \\
\hline & $(95 \% C l ; 1.7-7.2 \%)$ & $(95 \% C l ; 1.5-14.3 \%)$ & (95\%Cl; 3.6-13.9\%) \\
\hline
\end{tabular}

\section{Associations between food insecurity and socio- demographics}

There were no associations between socio-demographics including maternal education level and employment status with household or child food security in HEN or LEAD cohorts. Higher household crowding factors as indicated by more people sharing a bedroom or bathroom were associated with having less household and child food security in HEN but not LEAD cohorts (Rho $=0.29, p<0.01$ for number sharing a bedroom for total food security score and Rho $=0.28, p<0.01$ for number sharing a bath; Rho $=0.33, p<0.01$ for number sharing a bedroom for child food security score and Rho $=0.27, p<0.01$ for number sharing a bathroom).

Sociodemographic variables associated with poverty including not being employed and having a lower education level (high school versus any university or postgraduate training) were associated with increased levels of household and child food insecurity in TAB $(p<0.01)$. Furthermore, crowding metrics (persons/bedroom and persons/bathroom) were also significantly associated with household and child food insecurity in $\mathrm{TAB}(p<0.01)$. Additionally, being a preferred/dominant Spanish language speaker was also highly correlated with having less household and child food security in TAB $(\mathrm{z}=-6.8 p<0.01$ household security and $\mathrm{z}=-4.2$, $p<0.01$ for child security) but not in HEN or LEAD.

\section{Change from food insecurity in prior years}

Data from these same cohorts in the last year and prior years suggest that there is much more severe food insecurity with fewer families now being food secure during the COVID-19 pandemic. In LEAD interviews from 1 year ago, 76.9\% of families were household food secure and $87.9 \%$ had child food security. In the 6 months prior to COVID, we assessed a sub-sample of HEN families $(n=38)$ and $79.0 \%$ had household security and $89.2 \%$ had child food security. A larger study of HEN participants assessed more than 5 years ago $(n=$ 161) found that many of these families had struggled with food security in prior years with $56.6 \%$ with household food security and $69.3 \%$ with child food security.

\section{Association with COVID-19 infection}

There were 8 households surveyed in HEN that had COVID-19 infections as indicated by self-report positive reverse transcription polymerase chain reaction (RTPCR) tests out of the 111 total households surveyed that also had level of food security reported (7.2, 95\%CI; 3.6$13.9 \%$ ) (Table 2). Of these, $12.5 \%$ reported household food security compared with $34.7 \%$ of those households without individuals with COVID-19 positive members $(p=0.03)$. Adjusting for previously described crowding factors including persons per bedroom and bathroom, food insecurity category was not associated with COVID-19 positivity $(p=0.60)$. The percentage of COVID-19 positive households was lower among LEAD, $4.8 \%$ (95\%CI; $1.5-14.3 \%$ ); 3 out of 62 households having a COVID-19 infection (Table 2). There was no association between COVID-19 infection and household food insecurity in LEAD. Similarly, the infection rate in TAB household was lower than HEN (7 out of 200 or $3.5 \%$ 
(95\%CI; 1.7-7.2\%)) (Table 2). However, there was also a trend towards reduced food security among TAB households that were infected $(42.9 \%$ food secure among infected versus $60.6 \%$ of those uninfected and $28.6 \%$ had the highest level of food insecurity among infected compared with $5.2 \%$ of those uninfected, $p=0.02$ ). In a multivariable model of the TAB cohort adjusting for employment status, crowding as indicated by number of household members sharing a bathroom and Spanish language use, increased household food insecurity score trended towards a positive association with household COVID infection (OR 1.36, 95\%CI 0.97-1.92; $p=0.079$ ).

\section{Discussion}

Racial and ethnic minorities historically have worse outcomes during and after disasters as they already face a range of economic, political, and social barriers [23, 24]. These include but are not limited to racial discrimination, exploitation, limited English-speaking proficiency, and lack of legal status [24].

The overall prevalence of food insecurity in California between 2017 and 2019 was 9.9\% having low food security and $3.6 \%$ with very low food security [25]. Overall national levels of food insecurity were higher during this time period for Hispanic households (15.6\%).

Food insecurity has increased for American children and adults since the start of COVID-19 pandemic with elevations in every state [6]. Data from the Census Pulse Household Survey suggest that overall food insecurity tripled from 9.4 to $29.5 \%$ for households with children during COVID-19. Similarly, we report dramatic increases in food insecurity in our low income Latinx cohorts in the San Francisco Bay Area. These population groups already had low rates of food security. Prior to COVID-19, 76.9\% of LEAD families reported having food security but during COVID-19 only 38.1\% report food security. Similarly, HEN food security declined from $79.6 \%$ of adults having food security to $34.2 \%$. Decreases in child food security were also significant with 87.9\% secure prior to COVID declining $56.9 \%$ in LEAD and 89.2 to $54.1 \%$ in HEN. Our results in HEN and LEAD correspond with the high food insecurity rates (only $36 \%$ with food security) reported by US adults living $<250 \%$ below the federal poverty line during the COVID-19 pandemic by Wolfson and Leung (2020) [26].

Our findings of high food insecurity among three Latinx cohorts in the San Francisco Bay Area suggest rapid growth of food insecurity in urban areas impacted by COVID-19 shutdowns. Latinx individuals are among the most likely to be pushed into poverty as a result of pandemic-related unemployment [6, 27]. A significant percentage of our participants did not have any adult household members with employment at the time of the interview (more than one third of HEN and LEAD households and a quarter of TAB), and unemployment is strongly associated with food insecurity $[6,28]$. Additionally, Latinx individuals, such as the individuals in cohorts, who faced food insecurity prior to COVID-19 likely have more severe food insecurity during the pandemic and are particularly at risk for adverse outcomes [26].

Common coping strategies for food insecurity prior to COVID-19 include use of food pantries, borrowing money, going to homes of friends or family for meals, or sending children to relatives [29]. Some, if not all of these tactics to buffer families against food insecurity, are more limited given the recommended physical distancing measures being taken to prevent COVID-19 transmission [30]. Even when food pantry resources are available, Latinx families compared with other groups may face language barriers or concerns about legal status which limit use [31]. Shame can also result in people hiding hunger rather than going to food pantries and further increasing food vulnerability [32].

\section{Differences among Latinx cohorts}

There were large differences between our Latinx cohorts in levels of food insecurity. Part of this can be explained by socio-demographic differences by cohort. HEN and LEAD were recruited primarily at Zuckerberg San Francisco General Hospital, the local safety net county hospital with TAB recruited primarily at UCSF Benioff, a tertiary care academic hospital. The women recruited for HEN and LEAD cohorts had lower levels of eduation at recruitment (a low percentage reported any education beyond high school at recruitment (20.6\% for LEAD and $26.1 \%$ for $\mathrm{HEN}$ ) versus a much higher $65.7 \%$ for $\mathrm{TAB}$ ). Low education status is the greatest predictor of unemployment [33] and since the emergence of COVID19, employment losses are associated with low education levels [34]. Higher rates of household and child food insecurity in HEN and LEAD versus a much lower level in $\mathrm{TAB}$ (60\% with high food security) parallel the lower education levels and potential earning power in these cohorts [6, 28].

Additionally, over $90 \%$ of mothers from the HEN and LEAD were preferred/dominant Spanish-speakers while $65 \%$ of the TAB mothers were primary English-speaking. Being fluent in English leads to greater job opportunities [35] also potentially contributing to food insecurity $[6,28]$. Our previous studies with HEN and LEAD have also found that the majority of adults in these households are recent immigrants and foreign-born [17-19], potentially placing these families at a greater disadvantage in terms of accessing food and other government resources [36].

There was little difference in reported crowding indices (the number sharing bedrooms and bathrooms) 
between these three cohorts and although there were only associations between these metrics and food insecurity in TAB and HEN possibly based on differences in sample size (smaller sample size and less power in LEAD). Furthermore, we did not see any differences in food insecurity and education level or employment status for LEAD and HEN possibly because there was little variation in education level with the majority not having any advanced education beyond high school. We did not assess for underemployment common among Latinx adults but HEN and LEAD may have higher levels of underemployment [37]. Meanwhile for TAB, the association between education level and employment status and food insecurity was highly significant. Those with less education had greater food insecurity, possibly due to the heterogeneity in education levels for this cohort.

\section{Necessary interventions for Latinx families}

Our data suggest the urgent need for interventions for Latinx families with children, particularly those who are Spanish-speaking and with low education and employment levels in the context of the COVID-19 crisis. Data from our $\mathrm{TAB}$ cohort points to the heterogeneity among Latinx families in the Bay Area and the potential need for targeted interventions in specific communities. Due to urgent need, some safety net clinics in California have had creative responses in terms of bringing food to families. More funding and development of these efforts should be prioritized by state and county legislators $[38,39]$. Furthermore, individuals from underrepresented groups tend to not have enough accessible information in their preferred language and mode of communication [40]. For instance, $29 \%$ of Latinx in the United States have no English fluency [41]. There can also be distrust of the government and health agencies due to poor history and absence cultural competency $[36,40]$. The best implementations for COVID-19 relief will be made through the inclusivity of cultural richness, such as having information in multiple languages, modes of communication, and a diverse workforce to implement these interventions and work with diverse communities.

\section{Risk for COVID-19 infection with household food insecurity}

Previous researchers have noted an increased risk for COVID-19 morbidity and mortality due to low quality nutrition, obesity and associated metabolic disease [42]. While crowding factors and poverty are associated with risk for COVID-19 infection and food insecurity, food insecurity may be independently associated with increased risk for COVID-19 infection through poorer immune health and increased susceptibility to disease. Previous studies, particularly related to risk for HIV infection in the context of HIV exposure suggest that food insecurity may be an independent risk factor for infectious disease [15]. For example, other studies suggest that household food insecurity puts children in a more susceptible position for tuberculosis infection [43]. In multivariable analyses adjusting for crowding, unemployment and Spanish language use in the TAB cohort, food insecurity score trended towards association with household COVID infection (OR 1.25, 95\%CI 0.97-1.61). Further studies are needed with larger cohorts to assess the role between food insecurity, poor nutritional status and risk for infectious disease, inlcuding COVID-19.

\section{Conclusions and limitations}

Further population-based studies are needed with larger and heterogeneous cohorts to assess the impacts of COVID-19 in other high-risk population groups, in addition to Latinx. Rural communities and suburban areas may face different food insecurity stressors and as such our findings may not be relevant to rural or suburban areas. Government and non-governmental agencies need to prioritize poverty-alleviating measures including mitigating food insecurity as a means to address the public health demands of COVID-19 infection.

Lastly, while we focused on food insecurity, other studies suggest that the COVID-19 epidemic may have had broad ranging impacts of dietary practices. International studies suggest that the COVID-19 epidemic and the prolonged confinement and "stay at home measures" impacted children's dietary intake including increased intake of sweetened foods such as desserts [44]. Future studies should evaluate the impact of work from home policies and remote schooling on children's and family's dietary intake and nutritional health in addition to overall food security.

\section{Supplementary Information}

The online version contains supplementary material available at https://doi. org/10.1186/s40795-021-00419-1.

\section{Additional file 1.}

Acknowledgments

Thanks to all the participants who agreed to participate in our study.

\section{Authors' contributions}

JMW conceived of the study. ME, MRE and ADC collected data. JMW conducted data analysis. ME and ADC wrote the manuscript. SV provided comments on analysis. All authors approved the final version of the manuscript.

\section{Funding}

This project was supported by the generosity of the Eric and Wendy Schmidt by recommendation of the Schmidt Futures program, through Covid Catalyst at the Center of Emerging and Neglected Diseases. Additional funding came from NIH NIDDK 080825097458 as well as the Hellman Family Foundation and Allen Foundation and Marc and Lynn Benioff. 


\section{Availability of data and materials}

The data from this study is available on written request to the Principal Investigator (JMW).

\section{Declarations}

\section{Ethics approval and consent to participate}

The study was approved by the Committee on Human Research (CHR), the Institutional Review Board (IRB) of the University of California, San Francisco. All participants provided verbal consent for their participation and all aspects of the study were approved by the UCSF IRB (IRB numbers 11-06334; 1606163; 16-18535). All methods were conducted in accordance with the UCSF IRB approval.

\section{Consent for publication}

All authors have approved the final version of the manuscript for publication. All data is de-identified.

\section{Competing interests}

There are no competing interests.

\section{Received: 17 November 2020 Accepted: 12 March 2021} Published online: 11 June 2021

\section{References}

1. Lauren BN, Silver ER, Faye AS, Woo Baidal JA, Ozanne EM, Hur C. Predictors of household food insecurity in the United States during the COVID-19 pandemic. bioRxiv. 2020;2020(06):10.20122275. https://doi.org/10.1101/2020. 06.10.20122275.

2. Coleman-Jensen A, Rabbitt MP, Gregory CA, Singh A. Household food security in the United States in 2015. Usda.gov. https://www.ers.usda.gov/ webdocs/publications/79761/err215_summary.pdf?v=3774.6. Accessed September 23, 2020.

3. Coleman-Jensen A, Gregory C, Singh A. Household food security in the United States in 2013. SSRN Electron J. 2014. https://doi.org/10.2139/ssrn.2 504067.

4. Noe-Bustamante L, Hugo-Lopez M, Krogstad JM. US Hispanic Population Surpassed 60 Mission in 2019, but growth has slowed. Pew Res. 2109; https://www.pewresearch.org/fact-tank/2020/07/07/u-s-hispanic-populationsurpassed-60-million-in-2019-but-growth-has-slowed/.

5. Maani N, Galea S. COVID-19 and underinvestment in the health of the US population. Milbank Q. 2020;98(2):239-49. https://doi.org/10.1111/14680009.12462.

6. Schanzenbach D, Pitts A. How much has food insecurity risen? Evidence from the Census Household Pulse Survey [Institute for Policy Research (IPR) rapid research report]: Northwestern Institute for Policy Research; 2020. Accessed September 26, 2020. https://www.ipr.northwestern.edu/ documents/reports/ipr-rapid-research-reports-pulse-hh-data-10-june-2020. pdf

7. Hernandez DC, Reesor L, Murillo R. Gender disparities in the food insecurityoverweight and food insecurity-obesity paradox among low-income older adults. J Acad Nutr Diet. 2017;117(7):1087-96. https://doi.org/10.1016/j.jand.2 017.01.014.

8. Rabbitt M, Smith MD, Coleman-Jensen A. Food security among Hispanic adults in the United States, 2011-2014: United States Department of Agriculture, Economic Research Service; 2016.

9. Potochnick S, Arteaga I. A decade of analysis: household food insecurity among low-income immigrant children. J Fam Issues. 2018;39(2):527-51. https://doi.org/10.1177/0192513X16661216.

10. van Hook J, Balistreri KS. Ineligible parents, eligible children: food stamps receipt, allotments, and food insecurity among children of immigrants. Soc Sci Res. 2006;35(1):228-51. https://doi.org/10.1016/j.ssresearch.2004.09.001.

11. Clark E, Fredricks K, Woc-Colburn L, Bottazzi ME, Weatherhead J. Disproportionate impact of the COVID-19 pandemic on immigrant communities in the United States. PLoS Negl Trop Dis. 2020;14(7):e0008484. https://doi.org/10.1371/journal.pntd.0008484.

12. Bermudez E. As demand for food skyrockets due to coronavirus, food banks play catch-up: LA Times; 2020.

13. Bixler D, Miller AD, Mattison CP, Taylor B, Komatsu K, Peterson Pompa X et al. SARS-CoV-2-associated deaths among persons aged $<21$ years -
United States, February 12-July 31, 2020. MMWR Morb Mortal Wkly Rep. 2020;69(37):1324-9. https://doi.org/10.15585/mmwr.mm6937e4.

14. Nagata JM, Gomberg S, Hagan MJ, Heyman MB, Wojcicki JM. Food insecurity is associated with maternal depression and child pervasive developmental symptoms in low-income Latino households. J Hunger Environ Nutr. 2019;14(4):526-39. https://doi.org/10.1080/19320248.201 8.1434101.

15. Rollins N. Food insecurity-a risk factor for HIV infection. PLoS Med. 2007;(10): 1576-7. https://doi.org/10.1371/journal.pmed.0040301.

16. Kjaer TW, Faurholt-Jepsen D, Medrano R, Elwan D, Mehta K, Christensen VB, et al. Higher birthweight and maternal pre-pregnancy BMI persist with obesity association at age 9 in high risk Latino children. J Immigr Minor Health. 2019;21 (1):89-97. https://doi.org/10.1007/s10903-018-0702-0.

17. Wojcicki JM, Holbrook K, Lustig RH, Epel E, Caughey AB, Munoz RF, et al. Chronic Maternal Depression is Associated with Reduced Weight Gain in Latino Infants from Birth to 2 Years. PLoS One. 2011;6(2):e16737.

18. Ville AP, Heyman MB, Medrano R and JM Wojcicki. Early Antibiotic Exposure and Risk of Childhood Obesity in Latinos. Child Obes 2017; 13(2): doi: https://doi.org/10.1089/chi.2016.0235, 13, 3, 235.

19. Wojcicki JM, Medrano R, Lin J, Epel E. Increased cellular aging by 3 years of age in Latino, preschool children who consume more sugar-sweetened beverages. Child Obes. 2018;14(3):149-57. https://doi.org/10.1089/chi.2017. 0159.

20. USDA Economic Research Service. Survey Tools. 2020 Usda.gov. https:// www.ers.usda.gov/topics/food-nutrition-assistance/food-security-in-the-us/ survey-tools/. Accessed September 28, 2020.

21. Harrison GG, Stormer A, Herman DR, Winham DM. Development of a Spanish-Language Version of the US Household Food Security Survey Module. J Nutr. 133(4):1192-7.

22. Hromi-Fiedler A, Bermúdez-Millán A, Segura-Pérez S, Damio G, PérezEscamilla R. Adaptation of the U.S. food security survey module for lowincome pregnant Latinas: qualitative phase. J Hunger Environ Nutr. 2009; 4(1):62-80. https://doi.org/10.1080/19320240802706841.

23. Hutchins SS, Fiscella K, Levine RS, Ompad DC, McDonald M. Protection of racial/ethnic minority populations during an influenza pandemic. Am J Public Health. 2009:99(Suppl 2(S2)):S261-70.

24. Méndez M, Flores-Haro G, Zucker L. The (in) visible victims of disaster: understanding the vulnerability of undocumented Latino/a and indigenous immigrants. Geoforum. 2020;116:50-62. https://doi.org/10.1016/j.geoforum.2 020.07.007.

25. USDA Key statistics \& graphics, Food Security Status of US Households in 2019. Usda.gov. https://www.ers.usda.gov/topics/food-nutrition-assistance/ food-security-in-the-us/key-statistics-graphics.aspx. Accessed September 27, 2020.

26. Wolfson JA, Leung CW. Food insecurity and COVID-19: disparities in early effects for US adults. Nutrients. 2020;12(6):1648. https://doi.org/10.3390/nu12 061648.

27. Feeding America. Feedingamerica.org. The impact of the Coronavirus on food insecurity. https://www.feedingamerica.org/sites/default/files/2020-04/ Brief_Impact\%20of\%20Covid\%20on\%20Food\%20Insecurity\%204.22\%20\%2 8002\%29.pdf. Published 2020. Accessed September 27, 2020

28. Abrams SA, Avalos A, Gray M, Hawthorne KM. High level of food insecurity among families with children seeking routine care at federally qualified health centers during the coronavirus disease 2019 pandemic. J Pediatr X. 2020;4:100044.

29. Gundersen C, Ziliak JP. Food insecurity and health outcomes. Health Aff (Millwood). 2015;34(11):1830-9. https://doi.org/10.1377/hlthaff.2015.0645.

30. Wasserman D, van der Gaag R, Wise J. The term "physical distancing" is recommended rather than "social distancing" during the COVID-19 pandemic for reducing feelings of rejection among people with mental health problems. Eur Psychiatry. 2020;63(1):e52. https://doi.org/10.1192/j. eurpsy.2020.60.

31. Wright KE, Lucero J, Crosbie E. "It's nice to have a little bit of home, even if it's just on your plate" - perceived barriers for Latinos accessing food pantries. J Hunger Environ Nutr. 2020;15(4):496-513. https://doi.org/10.1 080/19320248.2019.1664963.

32. Zepeda L. Hiding hunger: food insecurity in middle America. Agric Hum Values. 2018;35(1):243-54. https://doi.org/10.1007/s10460-017-9818-4.

33. van Zon S, Reijneveld S. Mendes de Leon C, Bültmann U. the impact of low education and poor health on unemployment varies by work life stage. Int J Public Health. 2017;62(9):997-1006. https://doi.org/10.1007/500038-017-0972-7. 
34. Kochhar R. Hispanic women, immigrants, young adults, those with less education hit hardest by COVID-19 job losses. Pew Research Center. https:// www.pewresearch.org/fact-tank/2020/06/09/hispanic-women-immigrantsyoung-adults-those-with-less-education-hit-hardest-by-covid-19-job-losses/. Published 2020. Accessed September 28, 2020.

35. Park J. The earnings of immigrants in the United States: the effect of English-speaking ability. Am J Econ Sociol. 1999;58(1):43-56 https://wwwjstor-org.ucsf.idm.oclc.org/stable/3487874?seq=1\#metadata_info_tab_ contents. Accessed September 28, 2020.

36. Page KR, Venkataramani M, Beyrer C, Polk S. Undocumented US. immigrants and covid-19. N Engl J Med. 2020;382(21):e62.

37. Golden L And J Kim. Underemployment just Isn't working for US part-time workers. The Center for Law and Social Policy https://www.clasp.org/publica tions/report/brief/underemployment-just-isnt-working-us-part-time-workers, accessed October 4, 2020.

38. Ugwa-Oju D. How a pandemic exposed racial inequality, food insecurity in California's Central Valley. Fresno Bee https://www.fresnobee.com/fresnola nd/article243240666.html, accessed October 6, 2020.

39. Center for Care Innovations. LA Clinics Respond to Food Insecurity During COVID-19. https://www.careinnovations.org/resources/la-clinics-respond-tofood-insecurity-during-covid-19/, accessed October 6, 2020.

40. DeBruin D, Liaschenko J, Marshall MF. Social justice in pandemic preparedness. Am J Public Health. 2012;102(4):586-91. https://doi.org/10.21 05/AJPH.2011.300483.

41. United States Department of Health and Human Services. Office of Minority Health. Profile: Hispanic/Latino Americans. https://minorityhealth.hhs.gov/ omh/browse.aspx?lv|=3\&lvlid=64, Accessed October 11, 2020.

42. Belanger M, Hill M, Angelidi A, Dalamaga M, Sowers J, Mantzoros C. Covid19 and disparities in nutrition and obesity. N Engl J Med. 2020;383(11):e69. https://doi.org/10.1056/nejmp2021264.

43. Faust L, McCarthy a, Schreiber Y. Recommendations for the Screening of Latent Tuberculosis Infection in Indigenous Communities: A systematic Review of Screening Strategies Among High-Risk Groups in Low-Incidence Countries. BMC Public Health. 2018;198:979.

44. Ruiz-Roso MB, de Carvalho PP, Mantilla-Escalante DC, Ulloa N, Brun P, Acevedo-Correa D, et al. Covid-19 Confinement and Changes of Adolescent's Dietary Trends in Italy, Spain, Chile, Colombia and Brazil. Nutrients. 2020;12(6):1807. https://doi.org/10.3390/nu12061807.

\section{Publisher's Note}

Springer Nature remains neutral with regard to jurisdictional claims in published maps and institutional affiliations.

Ready to submit your research? Choose BMC and benefit from:

- fast, convenient online submission

- thorough peer review by experienced researchers in your field

- rapid publication on acceptance

- support for research data, including large and complex data types

- gold Open Access which fosters wider collaboration and increased citations

- maximum visibility for your research: over $100 \mathrm{M}$ website views per year

At $\mathrm{BMC}$, research is always in progress.

Learn more biomedcentral.com/submissions 